Microcirculation (2003) 10, 245-246

(1) 2003 Nature Publishing Group 1073-9688/03 \$25.00

www.nature.com/mn

\title{
Foreword
}

\section{Arrest, Migration, Survival and Proliferation of Leukocytes and Vascular Cells: The Many Faces of Chemokine Biology}

\author{
KLAUS LEY \\ University of Virginia Health System Cardiovascular Research Center, \\ Charlottesville, VA, USA \\ Microcirculation (2003) 10, 245-246 doi:10.1038/sj.mn.7800189
}

This issue of Microcirculation explores the role of chemokines, a family of small, secreted peptides that regulate leukocyte traffic in health and disease. Leading experts review the diverse function of these mediators, with an emphasis on their roles in microvascular physiology and pathophysiology. The most amazing aspect of this field of research is the diversity of functions that have been attributed to chemokines. Chemokines are classified according to their amino acid sequence as CC (no intervening amino acid between first two cysteinyl residues), CXC (one intervening amino acid), CX3C (three intervening amino acids), and $\mathrm{XC}$ (only one cysteinyl residue). They are numbered as CCL1-27, CXCL1-16, CX3CL1, and XCL1 $(10,11,15)$. They bind to at least $11 \mathrm{CC}$ receptors (CCR1-11), 6 CXC receptors (CXCR1-6), CX3CR1, and XCR1 $(10,11,15)$. Earlier notions that CXC chemokines attract granulocytes and $\mathrm{CC}$ receptors are responsible for mononuclear cell effects have been supplanted by more recent findings.

Chemokines may have emerged early in evolution from proteolytic fragments of tyrosyl transfer RNA synthetase, an essential intracellular enzyme, even before a circulatory system developed $(15,16)$. In complex mammalian organisms, chemokines not only regulate leukocyte development and chemotaxis, but also play important roles in their trafficking through the vascular and lymphatic systems. Some, but not all chemokines can be immobilized on the endothelial surface and can activate integrin adhesion receptors on rolling leukocytes to promote their arrest. Two reviews in this issue $(1,8)$ focus on the

For reprints of this article, contact Klaus Ley, University of Virginia Health System Cardiovascular Research Center, MR5 Building, Room 1013, PO Box 801394, Charlottesville, VA 22908, USA; e-mail klausley@virginia.edu.

Received 25 January 2003; accepted 25 January 2003 function of arrest chemokines, presenting different perspectives on how chemokine receptor signaling may be integrated with other signals in phagocytes (8) and how chemokine receptor engagement in lymphocytes will promote local integrin avidity upregulation in a fraction of a second (1).

One of the first chemokines discovered was CCL2 (monocyte chemoattractant protein-1), and it may well be one of the most important chemokines. The mice lacking CCL2 or its only known receptor, CCR2, show interesting and sometimes opposite phenotypes $(3,4)$. Although CCR2-deficient mice show a reduced T helper type 2 response, mice lacking CCL2 produce fewer T helper type 1 cytokines. The CCL2/CCR2 system seems to be a key regulator of inflammation in the lung (12) and other organs. Proinflammatory functions of chemokines also are addressed in several of the other articles.

Other well-known functions of chemokines include their role in regulating the trafficking of naïve and effector B-lymphocytes and T-lymphocytes. This trafficking function involves specialized microvessels, high endothelial venules, in lymph nodes, Peyer's patches, and other lymphatic organs. A review from Martin Lipp's group explores the impact of removing from mice either CCR7, the major chemokine receptor of naïve and some memory $\mathrm{T}$ cells, or CXCR5, a chemokine receptor on B cells and some B-helper T cells (9). In addition to these chemokines that play a role in many organs and tissues, recent research has identified some chemokines that are organ-specific. One review focuses on lymphocyte homing to the gut and gut-associated lymphatic tissues by engaging CCR6, CCR9, and CCR10 (7).

Recently, chemokines have been found to be involved in regulating angiogenesis $(2,13)$, largely in the context of inflammation. This new function is of particular interest to researchers studying the micro- 
circulation. The function of chemokines and chemokine receptors in platelets is a very novel aspect (5), as is the potential role of L-selectin in chemokinemediated arrest and migration (6). Recently, chemokine receptors have been found on endothelial and smooth muscle cells. These chemokine receptors include CCR5, CXCR4, and an unknown receptor for CCL2 (monocyte chemoattractant protein-1). Based on these findings, chemokine receptors are not only important in leukocytes, but possibly in all vascular cells, with implications for processes such as atherosclerosis and vascular injury (14).

The special issue of Microcirculation that is before you is not complete in the sense that it does not cover every aspect of chemokine biology. For example, homeostatic effects of chemokines like CXCL12 (stromal cell-derived factor-1) are not covered. Some chemokines like CXCL16, XCL1 (lymphotactin), or CX3CL1 (fractalkine), the functions of which are not well understood yet, are not discussed. Rather than being a comprehensive review, this special issue is aimed at highlighting some of the recent and exciting developments in this field that are so central to microvascular function. By necessity, the selection of themes flows from the areas of expertise of the authors.

\section{ACKNOWLEDGMENTS}

I wish to express sincere thanks to all contributors for volunteering their time, and to Lauren Vanderkuy for her editorial assistance.

\section{REFERENCES}

1. Alon R, Grabovsky V, Feigelson S. (2003). Chemokine induction of integrin avidity on rolling and arrested leukocytes: local signaling events or global stepwise activation? Microcirculation 10:297-311.

2. Buschman I, Heil M, Jost M, Schaper W. (2003). Influence of inflammatory cytokines on arteriogenesis. Microcirculation 10:371-379.

3. Charo IF, Peters W. (2003). Chemokine receptor 2 in atherosclerosis, infectious diseases and regulation of T cell polarization. Microcirculation 10:259-264.

4. Daly C, Rollins BJ. (2003). Monocyte chemoattractant protein-1 (CCL2) in inflammatory disease and adaptive immunity: therapeutic opportunities and controversies. Microcirculation 10:247-257.

5. Gear AR, Camerini D. (2003). Platelet chemokines and chemokine receptors: linking hemostasis, inflammation and host defense. Microcirculation 10:335350.

6. Khan A. Kubes P. (2003). L-selectin: an emerging player in chemokine biology. Microcirculation 10:351-358.

7. Kunkel EJ, Campbell DJ, Butcher EC. (2003). Chemokines in lymphocyte trafficking and intestinal immunity. Microcirculation 10:313-323.

8. Ley K. (2003). Arrest chemokines. Microcirculation 10:289-295.

9. Muller G, Lipp M. (2003). Shaping up adaptive immunity: the impact of CCR7 and CXCR5 on lymphocyte trafficking and homing. Microcirculation 10:325-334.

10. Murphy PM, Baggiolini M, Charo IF, Hebert CA, Horuk R, Matsushima K, Miller LH, Oppenheim JJ, Power CA. (2000). International Union of Pharmacology. XXII: nomenclature for chemokine receptors. Pharmacol Rev 52:145-176.

11. Olson TS, Ley K. (2002). Chemokines and chemokine receptors in leukocyte trafficking. Am J Physiol 283:R7-R28.

12. Rose CE, Sung SS, Fu SM. (2003). CCR2: increasing evidence for involvement in inflammatory disorders of the lung. Microcirculation 10:273-288.

13. Salcedo R, Oppenheim JJ. (2003). Role of chemokines in angiogenesis: CXCL12/SDF-1 and CXCR4 interaction, a key regulator of endothelial cell responses. Microcirculation 10:359-370.

14. Schecter AD, Berman AB, Taubman MA. (2003). Chemokine receptors in vascular smooth muscle. $\mathrm{Mi}$ crocirculation 10:265-272.

15. Slotnik A, Yoshie O. (2000). Chemokines: a new classification system and their role in immunity. Immunity 12:121-127.

16. Wakasugi K. Schimmel P. (1999). Two distinct cytokines released from a human aminoacyl-tRNA synthetase. Science 284:147-151. 